government's central motivation in 'going green' with the planted forest project in the first place.

Even so, the conservation project's organizers have a tough job. If the loggers or timber companies do not seem to be playing by the rules - for example, failing to protect conservation zones as promised, or not using low-impact logging techniques - they need to cry foul to the media or the appropriate government agency. This is the only way to ensure that the project pays more than lip service to its environmental goals. But given the delicate balance of conservation politics and economic goals in Sarawak, it will be difficult to do.

There are other ways to help keep the project on the right path. The conservation aspect depends on proving that there are species worth saving and finding ways to protect them. Ecologists and taxonomists from around the world can help by studying habitat, taking specimens and reporting back. Rather than popping in just to get samples for their collections, they should commit to periodic visits so that changes in both the natural forest and the plantation can be monitored systematically over time in different seasons. Many researchers have already started doing this, but more participation is needed to document the flora and fauna at stake.

One of the most creative proposals to make the most of the experiment in Sarawak concerns the tapir, a large-bodied, short-legged herbivore with splayed feet and a prehensile snout. There is archaeological evidence that Malayan tapirs (Tapirus indicus) were present on the island as recently as 60 years ago, and some researchers have argued that the conservation zone would be a perfect opportunity to bring them back. The mammal's significance in ecological terms might be small, but its restoration to its natural habitat could have considerable symbolic impact. Such reintroductions of large mammals are rare. Globally the Malayan tapir alternates between vulnerable and endangered on conservation lists. Its disappearance from Borneo was probably due to a loss of habitat following uncontrolled logging. What better way to show that logging is now controlled and its habitat protected?

Sadly, Sarawak's biodiversity programme will probably not be able to provide habitat for many other species that need it. Some will be lost. This is an inevitable outcome of a simple reality: to governments such as Sarawak's, as well as to the people who live in and around the forests, the use of the forest is a crucial part of their livelihoods.

The current project is ambitious and, as its organizers will readily admit, success is by no means assured. They haven't even agreed how success should be measured. But subject to these caveats, the project holds considerable promise as a model for biodiversity conservation in a southeast-Asian timber industry that badly needs one.

\section{South Africa's mentors}

\section{Announcing this year's Nature awards for scientific mentoring.}

N owhere is strong mentoring for young scientists more urgently required than in South Africa. The Centre for Research on Science and Technology at Stellenbosch University has shown that the country's research output declined in nominal terms between 1987 and 2001, and almost halved as a proportion of global scientific output, as measured by the production of articles listed by Thomson Scientific (ISI). South Africa's scientific workforce is ageing, with few young researchers being attracted into the system. In 1990, only $20 \%$ of peer-reviewed articles published in South Africa were written by people older than 50 , but this figure had risen to $49 \%$ by the year 2000 .

Additionally, there was no significant change in the demographic profile of publishing scientists between 1990 and 2000, with white scientists continuing to produce more than $90 \%$ of peer-reviewed articles. Women, who make up $40 \%$ of the academic staff at the country's universities, are responsible for authoring only $17 \%$ of articles. The National Research Foundation has attempted to address these problems by providing additional sources of funding to support black and female faculty members, and for black postgraduate students.

Originally the legacy of apartheid-based education, the dearth of black researchers has tragically continued into South Africa's democratic era. The post-apartheid government has been woefully unsuccessful in improving the maths and science education of black Africans since it took office in 1994: a 2004 report by the Johannesburg-based Centre for Development and Enterprise revealed that the same number of black school-leavers achieved maths grades sufficient for university entrance in science in 2002 as in 1991.

The country's universities are experiencing problems at two levels. First, far too few students - and particularly black students - enrol for undergraduate degrees in science, medicine and engineering. Second, relatively few students who complete first degrees continue their studies to doctoral level.

Whereas the former problem will require a long-term solution, the latter one could be solved relatively easily by substantially increasing the value of postgraduate bursaries. This is a measure the Department of Science and Technology is going to have to take if it is serious about its stated aim of increasing the number of doctoral graduates currently just over 1,100 annually - by an order of magnitude.

But numbers are not the whole story. Equally important for South Africa's future is the quality of science produced, and a critical factor here is the quality of mentoring that fledgling researchers receive. For all its problems, South Africa's universities include some with a strong scientific culture. There is no reason to suppose that, as a proportion of its academic population, there are fewer great science mentors in South Africa than anywhere else.

At Nature, we want to highlight them and the good things they do. Competitions for Nature's mentoring awards, initiated in 2005 and so far held in the United Kingdom and Australasia, are intended not only to celebrate the winners, but also to show the way for others. So we urge readers who know of outstanding mentors in South Africa to ensure that they are nominated. Two awards of 60,000 rand (US $\$ 8,300$ ) will be given: one for lifetime achievement and one for a track record in mid-career.

The closing date for nominations is 31 May. Details of the competition, the judges and how to nominate candidates for the awards can be found at www.nature.com/nature/mentoringawards/southafrica. 